
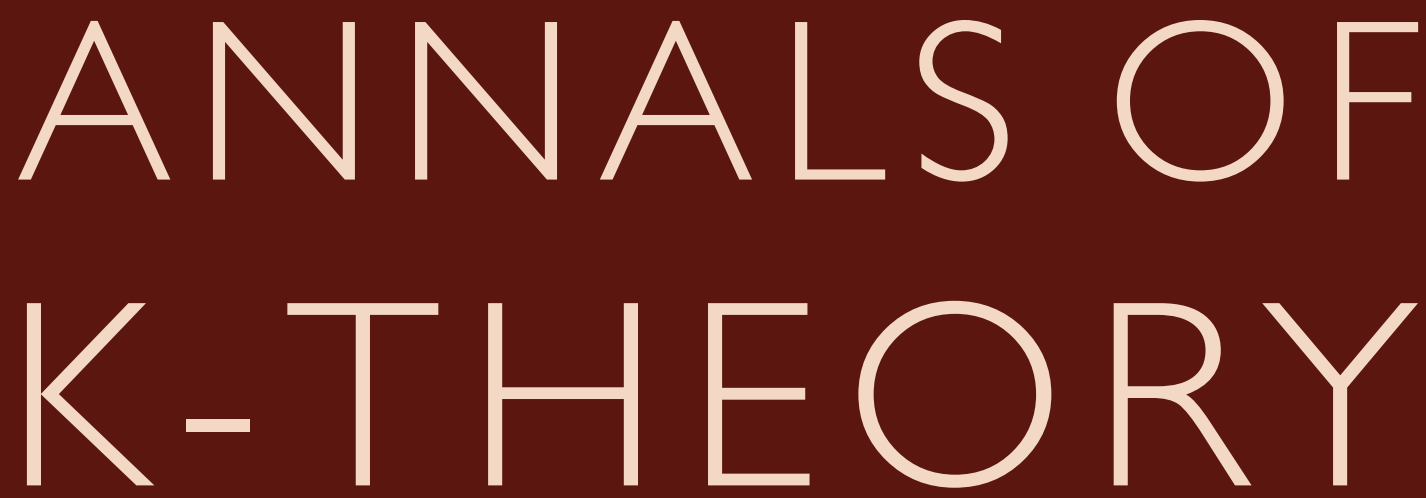

no. 1 vol 12016

On some negative motivic homology groups

Tohru Kohrita

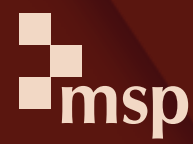
A JOURNAL OF THE K-THEORY FOUNDATION 


\title{
On some negative motivic homology groups
}

\author{
Tohru Kohrita
}

For an arbitrary separated scheme $X$ of finite type over a finite field $\mathbb{F}_{q}$ and a negative integer $j$, we prove, under the assumption of resolution of singularities, that $H_{-1}(X, \mathbb{Z}(j))$ is canonically isomorphic to $H_{-1}\left(\pi_{0}(X), \mathbb{Z}(j)\right)$ if $j=-1$ or -2 , and $H_{i}(X, \mathbb{Z}(j))$ vanishes if $i \leq-2$ and $i-j \leq 1$. As the group $H_{-1}\left(\pi_{0}(X), \mathbb{Z}(j)\right)$ is explicitly known, this gives a explicit calculation of motivic homology of degree -1 and weight -1 or -2 of an arbitrary scheme over a finite field.

\section{Introduction}

In this paper, we assume that schemes are separated and of finite type over a perfect field. The finite field with $q$ elements is written as $\mathbb{F}_{q}$. For a scheme $X, \pi_{0}(X)$ denotes the spectrum of $O_{X}(X)^{\text {ét }}$, the largest étale $k$-algebra contained in $\mathscr{O}_{X}(X)$ that is finite over $k$. The properties of $\pi_{0}(X)$ relevant to us can be found in [Liu 2002, pp. 495-496].

The aim of this paper is to prove the following theorem on motivic homology.

Theorem 1.1. Assume that resolution of singularities holds over $\mathbb{F}_{q}$. Let $i$ and $j$ be negative integers. Then, for all schemes $X$ over $\mathbb{F}_{q}$,

$$
H_{i}(X, \mathbb{Z}(j))=0
$$

if $i \leq-2$ and $i-j \leq 1$. In degree $i=-1$, the canonical map

$$
\alpha_{X}: H_{-1}(X, \mathbb{Z}(j)) \longrightarrow H_{-1}\left(\pi_{0}(X), \mathbb{Z}(j)\right)
$$

is an isomorphism if $i-j \leq 1$, i.e., $j=-1$ or -2 .

Since $\pi_{0}(X)$ is finite étale over $\mathbb{F}_{q}$, it is a finite disjoint union of spectra of finite fields. Hence, the isomorphism $H_{-1}(X, \mathbb{Z}(j)) \cong H_{-1}\left(\pi_{0}(X), \mathbb{Z}(j)\right)$ of Theorem 1.1 and the explicit computation of negative motivic homology groups of finite fields as in Lemma 2.1 give an explicit computation of $H_{-1}(X, \mathbb{Z}(j))(j=-1$ or -2$)$ for an arbitrary scheme $X$ over $\mathbb{F}_{q}$. In particular, if $X$ is geometrically connected over $\mathbb{F}_{q}$ (this is equivalent to requiring that $X$ be connected and $\pi_{0}(X)=\operatorname{Spec} \mathbb{F}_{q}$ [Liu 2002, Chapter 10, Corollary 2.21(a)]), we have the following corollary.

MSC2010: primary 14F42; secondary 19E15.

Keywords: motivic homology, schemes over finite fields. 
Corollary 1.2. Under resolution of singularities, if $X$ is a geometrically connected scheme over $\mathbb{F}_{q}$ and $j=-1$ or -2 , there is a canonical isomorphism

$$
H_{-1}(X, \mathbb{Z}(j)) \stackrel{\sim}{\longrightarrow} H_{-1}\left(\operatorname{Spec} \mathbb{F}_{q}, \mathbb{Z}(j)\right) \cong \mathbb{F}_{q^{-j}}^{\times} .
$$

Remark 1.3. It is worth noting that if one assumes Parshin's conjecture the statement in Theorem 1.1 holds for all negative integers $i$ and $j$ without the bound $i-j \leq 1$. One only needs to invoke [Kondo and Yasuda 2013, Proposition 4.1] instead of Proposition 2.7 in order to prove the claims of Lemma 4.1, Proposition 4.2 and Proposition 4.3 without the bounding conditions on $i-j$. One may similarly prove Proposition 4.7, Lemma 4.9 and Lemma 4.10 for all negative integers $j$.

Theorem 1.1 is a version in the context of motivic homology of the following theorem of Kondo and Yasuda on Borel-Moore motivic homology. In fact, if the scheme $X$ is proper, our Theorem 1.1 is due to Kondo and Yasuda:

Theorem 1.4 [Kondo and Yasuda 2013, Theorem 1.1]. Let $j=-1$ or -2 and let $X$ be a connected scheme over a finite field $\mathbb{F}_{q}$. If $X$ is not proper,

$$
H_{-1}^{\mathrm{BM}}(X, \mathbb{Z}(j))=0 .
$$

If $X$ is proper, the pushforward maps

$$
H_{-1}^{\mathrm{BM}}(X, \mathbb{Z}(j)) \longrightarrow H_{-1}^{\mathrm{BM}}\left(\operatorname{Spec} \mathrm{O}_{X}(X), \mathbb{Z}(j)\right)
$$

are isomorphisms.

Theorem 1.4 itself is a generalization of [Akhtar 2004, Proposition 3.1], where the claim for $j=-1$ was proved for smooth projective schemes $X$.

The case $i \leq-2$ of our Theorem 1.1 is also due to Kondo and Yasuda if the scheme $X$ is proper (see Proposition 2.7).

Theorem 1.1 and Theorem 1.4 are related as follows. If $X$ is a scheme over $\mathbb{F}_{q}$, Spec $\mathrm{O}_{X}(X)$ is also a scheme over $\mathbb{F}_{q}$. (Recall our convention on schemes.) Thus, the canonical factorization

$$
X \longrightarrow \operatorname{Spec}_{X}(X) \longrightarrow \pi_{0}(X) \longrightarrow \operatorname{Spec} \mathbb{F}_{q}
$$

of the structure morphism of $X$ gives, on applying Theorem 1.1, the isomorphisms

$$
H_{i}(X, \mathbb{Z}(j)) \stackrel{\sim}{\longrightarrow} H_{i}\left(\operatorname{Spec} \mathscr{O}_{X}(X), \mathbb{Z}(j)\right) \stackrel{\sim}{\rightarrow} H_{i}\left(\pi_{0}(X), \mathbb{Z}(j)\right)
$$

because $\pi_{0}\left(\operatorname{Spec} O_{X}(X)\right)=\pi_{0}(X)$ by definition. It is trivially true that the first map is an isomorphism if $X$ is affine and so is the second if $X$ is proper. Since motivic homology and Borel-Moore homology agree for proper schemes, the theorem of Kondo and Yasuda says that the first map is an isomorphism when $X$ is proper. They proved this without assuming resolution of singularities. Our Theorem 1.1 
claims that both maps are always isomorphisms if we assume the existence of resolution of singularities.

Let us end this introduction with a summary of the properties of motivic homology and cohomology theories which we shall use freely in the subsequent sections.

By motivic (co)homology (with compact supports) with coefficients in an abelian group $A$, we mean the following four theories defined for schemes $X$ over a perfect field $k$ :

- motivic homology,

$$
H_{i}(X, A(j)):=\operatorname{Hom}_{\mathrm{DM}_{\mathrm{Nis}}^{-}(k)}(A(j)[i], M(X)) ;
$$

- motivic cohomology,

$$
H^{i}(X, A(j)):=\operatorname{Hom}_{\mathrm{DM}_{\mathrm{Nis}}^{-}(k)}(M(X), A(j)[i]) ;
$$

- motivic homology with compact supports,

$$
H_{i}^{\mathrm{BM}}(X, A(j)):=\mathrm{CH}_{j}(X, i-2 j ; A) ;
$$

- motivic cohomology with compact supports,

$$
H_{c}^{i}(X, A(j)):=\operatorname{Hom}_{\mathrm{DM}_{\mathrm{Nis}}^{-}(k)}\left(M^{c}(X), A(j)[i]\right) .
$$

Here $\mathrm{DM}_{\mathrm{Nis}}^{-}(k)$ is Voevodsky's triangulated category of motives [Voevodsky et al. 2000, Chapter 5] and $\mathrm{CH}_{j}(X, i-2 j ; A)$ is Bloch's [1986] higher Chow group. We refer to motivic homology with compact supports as Borel-Moore homology. We index higher Chow groups "homologically", by dimension of cycles, contrary to the more common indexing by codimension of cycles. With this indexing $\mathrm{CH}_{r}(X, s ; A)$ is a subquotient of the group of cycles of dimension $r+s$ in $X \times \Delta^{s}$ that intersect properly with all faces. The advantage of this convention is that we do not need to require $X$ to be equidimensional. If $X$ is equidimensional, we have $\mathrm{CH}_{r}(X, s ; A)=\mathrm{CH}^{\operatorname{dim} X-r}(X, s ; A)$.

There is a canonical isomorphism [Voevodsky et al. 2000, Chapter 5, Proposition 4.2.9; Mazza et al. 2006, Proposition 19.18]

$$
\operatorname{Hom}_{\mathrm{DM}_{\mathrm{Nis}}^{-}(k)}\left(\mathbb{Z}(j)[i], M^{c}(X)\right) \stackrel{\sim}{\longrightarrow} \mathrm{CH}_{j}(X, i-2 j)
$$

if $X$ is quasiprojective and $k$ admits resolution of singularities in the sense of [Voevodsky et al. 2000, Chapter 4, Definition 3.4]. We chose to define Borel-Moore homology by Bloch's higher Chow groups mainly because they have localization sequences without assuming resolution of singularities. For motivic cohomology, it is known that if $X$ is smooth and of pure dimension $d$, there is a canonical isomorphism [Mazza et al. 2006, Theorem 19.1]

$$
H^{i}(X, A(j)) \stackrel{\sim}{\longrightarrow} \mathrm{CH}_{d-j}(X, 2 j-i ; A) \stackrel{\text { def }}{=} H_{2 d-i}^{\mathrm{BM}}(X, A(d-j)) .
$$


The theories with and without compact supports agree for proper schemes, as the canonical morphism $M(X) \rightarrow M^{c}(X)$ becomes the identity. Moreover, if $X$ is a smooth scheme of pure dimension $d$ and $k$ admits resolution of singularities, there is a canonical isomorphism [Voevodsky et al. 2000, Chapter 5, Theorem 4.3.7(3)]

$$
H_{i}(X, \mathbb{Z}(j)) \cong H_{c}^{2 d-i}(X, \mathbb{Z}(d-j)) .
$$

\section{Borel-Moore homology}

In this preliminary section, we review some results on Borel-Moore homology groups. Let us begin with an explicit computation of all negative motivic homology groups of a finite field, following [Kondo and Yasuda 2013, Remark 2.6].

Lemma 2.1. Let $i$ and $j$ be negative integers. Then

$$
H_{i}\left(\operatorname{Spec} \mathbb{F}_{q}, \mathbb{Z}(j)\right)= \begin{cases}\mathbb{F}_{q^{-j}} & \text { if } i=-1, \\ 0 & \text { otherwise. }\end{cases}
$$

Proof. For $i<j \leq-1$,

$$
H_{i}\left(\operatorname{Spec} \mathbb{F}_{q}, \mathbb{Z}(j)\right) \cong \mathrm{CH}_{j}\left(\operatorname{Spec} \mathbb{F}_{q}, i-2 j\right)=0
$$

for dimension reasons. For $j \leq i$, consider the long exact sequence

$\cdots \rightarrow H^{-i-1}\left(\operatorname{Spec} \mathbb{F}_{q}, \mathbb{Q}(-j)\right) \rightarrow H^{-i-1}\left(\operatorname{Spec} \mathbb{F}_{q}, \mathbb{Q} / \mathbb{Z}(-j)\right)$

$$
\rightarrow H^{-i}\left(\operatorname{Spec} \mathbb{F}_{q}, \mathbb{Z}(-j)\right) \rightarrow H^{-i}\left(\operatorname{Spec} \mathbb{F}_{q}, \mathbb{Q}(-j)\right) \rightarrow \cdots .
$$

The first and the last terms vanish because

$$
H^{t}\left(\operatorname{Spec} \mathbb{F}_{q}, \mathbb{Q}(-j)\right)=\mathrm{CH}_{j}\left(\mathbb{F}_{q},-t-2 j\right)_{\mathbb{Q}} \hookrightarrow K_{-t-2 j}\left(\mathbb{F}_{q}\right)_{\mathbb{Q}}=0
$$

if $-t-2 j \neq 0$. The embedding follows from Bloch's Riemann-Roch theorem [1986, Theorem 9.1] and the last equality by Quillen's calculation [1972, Theorem 8] of $K$-groups of finite fields. Now, because we are in the range $-i-1<-j$, we may apply [Geisser and Levine 2000, Theorem 8.4] and [Geisser and Levine 2001, Corollary 1.2], whose hypotheses are satisfied by the theorem of Rost and Voevodsky, to obtain

$$
\begin{aligned}
H_{i}\left(\operatorname{Spec} \mathbb{F}_{q}, \mathbb{Z}(j)\right) & \cong H^{-i}\left(\operatorname{Spec} \mathbb{F}_{q}, \mathbb{Z}(-j)\right) \\
& \cong H^{-i-1}\left(\operatorname{Spec} \mathbb{F}_{q}, \mathbb{Q} / \mathbb{Z}(-j)\right) \\
& \cong \bigoplus_{l} H^{-i-1}\left(\operatorname{Spec} \mathbb{F}_{q}, \mathbb{Q}_{l} / \mathbb{Z}_{l}(-j)\right) \\
& \cong \bigoplus_{l \neq p} H_{\text {êt }}^{-i-1}\left(\operatorname{Spec} \mathbb{F}_{q}, \mathbb{Q}_{l} / \mathbb{Z}_{l}(-j)\right) .
\end{aligned}
$$


Hence, if $i \leq-3$,

$$
H_{i}\left(\operatorname{Spec} \mathbb{F}_{q}, \mathbb{Z}(j)\right) \cong \bigoplus_{l \neq p} H_{\text {êt }}^{-i-1}\left(\operatorname{Spec} \mathbb{F}_{q}, \mathbb{Q}_{l} / \mathbb{Z}_{l}(-j)\right)=0,
$$

for $\mathbb{F}_{q}$ has cohomological dimension 1 .

If $i=-1$,

$$
\begin{aligned}
H_{-1}\left(\operatorname{Spec} \mathbb{F}_{q}, \mathbb{Z}(j)\right) & \cong \bigoplus_{l \neq p} H_{\mathrm{et}}^{0}\left(\operatorname{Spec} \mathbb{F}_{q}, \mathbb{Q}_{l} / \mathbb{Z}_{l}(-j)\right) \\
& \cong \bigoplus_{l \neq p} \mathbb{Z} / l^{r_{l}} \cong \mathbb{Z} /\left(q^{-j}-1\right) \cong \mathbb{F}_{q^{-j}}^{\times},
\end{aligned}
$$

where $r_{l}$ is the number such that $q^{-j}-1=\prod_{l} l^{r_{l}}$.

Finally, for $i=-2$, we need to show that the group $H_{\text {et }}^{1}\left(\operatorname{Spec} \mathbb{F}_{q}, \mathbb{Q}_{l} / \mathbb{Z}_{l}(-j)\right)$ vanishes for an arbitrary prime $l \neq p$. Since this is a Galois cohomology group of a finite field with torsion coefficients, there is an exact sequence

$$
\begin{aligned}
& 0 \rightarrow H_{\text {ét }}^{0}\left(\operatorname{Spec} \mathbb{F}_{q}, \mathbb{Q}_{l} / \mathbb{Z}_{l}(-j)\right) \rightarrow \mathbb{Q}_{l} / \mathbb{Z}_{l}(-j) \\
& \stackrel{\text { id }- \text { Frob }}{\longrightarrow} \mathbb{Q}_{l} / \mathbb{Z}_{l}(-j) \rightarrow H_{\text {ét }}^{1}\left(\operatorname{Spec} \mathbb{F}_{q}, \mathbb{Q}_{l} / \mathbb{Z}_{l}(-j)\right) \rightarrow 0 .
\end{aligned}
$$

Since $\mathbb{Q}_{l} / \mathbb{Z}_{l}(-j)$ is a divisible group, one can easily see that the homomorphism id - Frob is either zero or surjective. As we have seen above, the first term of this exact sequence is a finite group. Thus, id - Frob is not the zero map, so it must be surjective. This shows the vanishing of $H_{\text {ét }}^{1}\left(\operatorname{Spec} \mathbb{F}_{q}, \mathbb{Q}_{l} / \mathbb{Z}_{l}(-j)\right)$.

Remark 2.2. Since motivic homology is defined for schemes of finite type over some base field, for Lemma 2.1 to make sense, we need to specify the base field of Spec $\mathbb{F}_{q}$. However, as the proof shows, the lemma holds for any choice of the base field. More generally, see Lemma 4.6.

Let us further evaluate other relevant motivic invariants for later use.

Lemma 2.3. (i) $H^{i}\left(\operatorname{Spec} \mathbb{F}_{q}, \mathbb{Q}(j)\right)=0$ unless $(i, j)=(0,0)$.

(ii) If $K$ is a finitely generated field of transcendence degree 1 over $\mathbb{F}_{q}$, then $H^{i}(\operatorname{Spec} K, \mathbb{Q}(j))=0$ unless $(i, j)=(0,0)$ or $(1,1)$.

Proof. (i) For dimension reasons, the cohomology group in question vanishes unless $0 \leq j$ and $i \leq j$. By Bloch's Riemann-Roch theorem [1986, Theorem 9.1], there is an embedding $H^{i}\left(\operatorname{Spec} \mathbb{F}_{q}, \mathbb{Q}(j)\right) \hookrightarrow K_{-i+2 j}\left(\mathbb{F}_{q}\right)_{\mathbb{Q}}$. Since the positive degree $K$-groups of a finite field are torsion [Quillen 1972, Theorem 8], this implies $H^{i}\left(\operatorname{Spec} \mathbb{F}_{q}, \mathbb{Q}(j)\right)=0$ when $-i+2 j \geq 1$. Hence, the group $H^{i}\left(\operatorname{Spec} \mathbb{F}_{q}, \mathbb{Q}(j)\right)$ vanishes unless $0 \leq j, i \leq j$ and $-i+2 j \leq 0$, i.e., $(i, j)=(0,0)$. 
(ii) Let us first note that, for dimension reasons, the cohomology group in question vanishes unless $i \leq j$ and $j \geq 0$.

Let $X$ be a smooth projective curve over $\mathbb{F}_{q}$ with function field $K$. For a nonempty open subscheme $U$ of $X$, the localization sequence for higher Chow groups yields an exact sequence (because $X, U$ and $X \backslash U$ are all smooth) $H^{i-2}(X \backslash U, \mathbb{Q}(j-1)) \rightarrow H^{i}(X, \mathbb{Q}(j)) \rightarrow H^{i}(U, \mathbb{Q}(j)) \rightarrow H^{i-1}(X \backslash U, \mathbb{Q}(j-1))$.

If $j \neq 1$ or $i<j=1$, the above two cohomology groups of $X \backslash U$ vanish by (i). Thus we obtain an isomorphism

$$
H^{i}(X, \mathbb{Q}(j)) \stackrel{\sim}{\longrightarrow} H^{i}(U, \mathbb{Q}(j)) .
$$

Taking the colimit over nonempty open subschemes $U$ of $X$ and applying [Mazza et al. 2006, Lemma 3.9], we obtain an isomorphism

$$
H^{i}(X, \mathbb{Q}(j)) \stackrel{\sim}{\longrightarrow} H^{i}(\operatorname{Spec} K, \mathbb{Q}(j)) .
$$

By Bloch's Riemann-Roch theorem, $H^{i}(X, \mathbb{Q}(j))$ is a subgroup of $K_{-i+2 j}(X)_{\mathbb{Q}}$, and this group vanishes if $-i+2 j>0$, by Harder's theorem [1977]. This means that $H^{i}(\operatorname{Spec} K, \mathbb{Q}(j))=0$ unless $(i, j)=(0,0)$ or $(1,1)$.

Lemma 2.5. Let $X$ be a smooth curve over $\mathbb{F}_{q}$. Then $H^{i}(X, \mathbb{Q}(j))=0$ unless $(i, j)=(0,0),(1,1)$ or $(2,1)$.

Proof. Let $K$ be the function field of $X$. By the same argument used to construct the isomorphism (2.4) in the proof of Lemma 2.3(ii), we obtain for an arbitrary smooth curve $X$ a canonical isomorphism

$$
H^{i}(X, \mathbb{Q}(j)) \stackrel{\sim}{\longrightarrow} H^{i}(\operatorname{Spec} K, \mathbb{Q}(j))
$$

if $j \neq 1$ or $j=1$ but $i \neq 1,2$.

Thus, by Lemma 2.3(ii), $H^{i}(X, \mathbb{Q}(j))=0$ when $j \neq 1$ unless $(i, j)=(0,0)$, and $H^{i}(X, \mathbb{Q}(j))$ also vanishes when $j=1$ and $i \neq 1,2$. Hence, the lemma follows.

The next two lemmas are special cases of [Kondo and Yasuda 2013, Proposition 4.1, Lemma 4.2]. In that paper, a more general claim is proved under the assumption of Parshin's conjecture. The lemmas below are the part where Parshin's conjecture is not necessary. We include their proofs for the convenience of the reader.

Lemma 2.6 [Kondo and Yasuda 2013, Lemma 4.2]. Let X be an irreducible scheme of dimension $d \geq 1$ over $\mathbb{F}_{q}$. Then, for $i, j \leq-1$ with $i-j \leq 1$,

$$
\operatorname{colim}_{U} H_{i}^{\mathrm{BM}}(U, \mathbb{Z}(j))=0,
$$

where $U$ runs through the set of all nonempty open subschemes of $X$. 
Proof. Let $K$ denote the function field of $X$. By definition, we have $\operatorname{colim}_{U} H_{i}^{\mathrm{BM}}(U, \mathbb{Z}(j))=\operatorname{colim}_{U} \mathrm{CH}^{d-j}(U, i-2 j) \cong \mathrm{CH}^{d-j}(\operatorname{Spec} K, i-2 j)$.

Hence, for dimension reasons, $\operatorname{colim}_{U} H_{i}^{\mathrm{BM}}(U, \mathbb{Z}(j))=0$ if $d-j>i-2 j$, i.e., $d>i-j$.

It remains to prove that $\mathrm{CH}^{d-j}(\operatorname{Spec} K, i-2 j)=0$ for $d \leq i-j$, i.e., when $d=1$. In this case, observe that we have the equality $i=j+1$ and the inequality $j=i-1 \leq-2$. Hence,

$$
\mathrm{CH}^{1-j}(\operatorname{Spec} K, i-2 j)=\mathrm{CH}^{1-j}(\operatorname{Spec} K, 1-j) \cong K_{1-j}^{M}(K),
$$

but the last group vanishes by the calculation of Milnor $K$-groups of degree $\geq 3$ of a global field [Bass and Tate 1973, II, Theorem 2.1(3)].

Proposition 2.7 [Kondo and Yasuda 2013, Proposition 4.1]. Let $X$ be a scheme over $\mathbb{F}_{q}$. Then we have $H_{i}^{\mathrm{BM}}(X, \mathbb{Z}(j))=0$ if $i \leq-2, j \leq-1$ and $i-j \leq 1$.

Proof. We prove this by induction on the dimension of $X$. We may suppose that $X$ is a reduced scheme because the Borel-Moore homology groups of a scheme and its reduction are the same. Thus, when $\operatorname{dim} X=0$, it is enough to show the claim for Spec $\mathbb{F}_{q^{n}}$. This case was treated in Lemma 2.1 and Remark 2.2.

Suppose, now, that the proposition is true for dimension $\leq d-1$. Let us first prove the claim for an irreducible scheme $X$ of dimension $d$. The localization sequence for Borel-Moore homology gives the exact sequence

$$
\operatorname{colim}_{Y} H_{i}^{\mathrm{BM}}(Y, \mathbb{Z}(j)) \longrightarrow H_{i}^{\mathrm{BM}}(X, \mathbb{Z}(j)) \longrightarrow \operatorname{colim}_{Y} H_{i}^{\mathrm{BM}}(X \backslash Y, \mathbb{Z}(j)),
$$

where $Y$ runs through the set of reduced closed subschemes of $X$ whose underlying sets are proper subsets of that of $X$. Since the first term is zero by the induction hypothesis and the last vanishes by Lemma 2.6, we obtain $H_{i}^{\mathrm{BM}}(X, \mathbb{Z}(j))=0$.

Now, for a general $X$, consider the abstract blowup

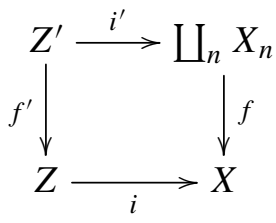

where the $X_{n}$ are the irreducible components of $X$ and $Z$ is the reduced closed subscheme of $X$ where $f$ is not an isomorphism. This gives rise to an exact sequence $H_{i}^{\mathrm{BM}}(Z, \mathbb{Z}(j)) \oplus \bigoplus_{n} H_{i}^{\mathrm{BM}}\left(X_{n}, \mathbb{Z}(j)\right) \stackrel{i_{*}-f_{*}}{\longrightarrow} H_{i}^{\mathrm{BM}}(X, \mathbb{Z}(j)) \stackrel{\delta}{\longrightarrow} H_{i-1}^{\mathrm{BM}}\left(Z^{\prime}, \mathbb{Z}(j)\right)$,

where $\delta$ denotes the connecting map. Hence, the vanishing of $H_{i}^{\mathrm{BM}}(X, \mathbb{Z}(j))$ follows from the induction hypothesis and the case of irreducible schemes. 


\section{With $\mathbb{Q}$-coefficients}

Before proving Theorem 1.1 in Section 4 under the assumption of resolution of singularities, we shall prove a weaker but unconditional result without assuming any conjectures. We use de Jong's alteration [1996] in place of smooth compactification and use results of Kelly [2012], in particular the existence of a localization sequence for motivic cohomology with compact supports with $\mathbb{Z}[1 / p]$-coefficients.

Theorem 3.1. For a smooth scheme $X$ over $\mathbb{F}_{q}$ and $i, j \leq-1$ with $i-j \leq 1$,

$$
H_{i}(X, \mathbb{Q}(j))=0 \text {. }
$$

We need a lemma.

Lemma 3.2. Suppose $X$ is a scheme over $\mathbb{F}_{q}$ of dimension at most $d-1$. Then

$$
H_{c}^{2 d-i}(X, \mathbb{Q}(d-j))=0
$$

if $i, j \leq 0$ and $i-j \leq 2$.

Proof. First, observe that the result is true for all $d \geq 1$ if $\operatorname{dim} X=0$, by Lemma 2.3(i).

We shall prove the lemma for a fixed $d=d_{0}$ by induction on $\operatorname{dim} X$. In doing so, we may suppose that the lemma is true for $d \leq d_{0}-1$ (by induction on $d$ ). Suppose that the lemma is true for schemes of dimension $\leq n-1$, and let $X$ be a scheme of dimension $n$. There is a localization sequence [Kelly 2012, Proposition 5.5.5] $H_{c}^{2 d_{0}-i}\left(X_{\mathrm{sm}}, \mathbb{Q}\left(d_{0}-j\right)\right) \rightarrow H_{c}^{2 d_{0}-i}\left(X, \mathbb{Q}\left(d_{0}-j\right)\right) \rightarrow H_{c}^{2 d_{0}-i}\left(X \backslash X_{\mathrm{sm}}, \mathbb{Q}\left(d_{0}-j\right)\right)$,

where $X_{\mathrm{sm}}$ is the smooth locus of $X$. The last term vanishes by the induction hypothesis. As for the first term, if we write $X_{\mathrm{sm}}=\bigsqcup X_{i}$ (where the $X_{i}$ are the connected components of $X$ ), we have

$$
H_{c}^{2 d_{0}-i}\left(X_{\mathrm{sm}}, \mathbb{Q}\left(d_{0}-j\right)\right) \cong \bigoplus_{i} H_{c}^{2 d_{0}-i}\left(X_{i}, \mathbb{Q}\left(d_{0}-j\right)\right) .
$$

So, in order to show that the first term is zero, it suffices to show that

$$
H_{c}^{2 d_{0}-i}\left(X, \mathbb{Q}\left(d_{0}-j\right)\right)=0
$$

for a smooth integral scheme $X$ of dimension $n$. (For schemes of smaller dimensions, the vanishing statement follows from the induction hypothesis.)

Now, let $U$ be a nonempty open subscheme of $X$, and consider the localization sequence [Kelly 2012, Proposition 5.5.5]

$$
\begin{aligned}
\cdots \rightarrow H_{c}^{2 d_{0}-i-1}\left(X \backslash U, \mathbb{Q}\left(d_{0}-j\right)\right) \rightarrow H_{c}^{2 d_{0}-i}\left(U, \mathbb{Q}\left(d_{0}-j\right)\right) \\
\rightarrow H_{c}^{2 d_{0}-i}\left(X, \mathbb{Q}\left(d_{0}-j\right)\right) \rightarrow H_{c}^{2 d_{0}-i}\left(X \backslash U, \mathbb{Q}\left(d_{0}-j\right)\right) \rightarrow \cdots .
\end{aligned}
$$


The last term vanishes by the induction hypothesis, and the first term too since the lemma is known for $d=d_{0}-1$ and since

$$
H_{c}^{2 d_{0}-i-1}\left(X \backslash U, \mathbb{Q}\left(d_{0}-j\right)\right)=H_{c}^{2\left(d_{0}-1\right)-(i-1)}\left(X \backslash U, \mathbb{Q}\left(\left(d_{0}-1\right)-(j-1)\right)\right) .
$$

Thus, we see that there is an isomorphism

$$
H_{c}^{2 d_{0}-i}\left(U, \mathbb{Q}\left(d_{0}-j\right)\right) \stackrel{\sim}{\longrightarrow} H_{c}^{2 d_{0}-i}\left(X, \mathbb{Q}\left(d_{0}-j\right)\right) .
$$

This means that in order to show the claim for $X$ it is enough to prove it for some open subscheme $U$ of $X$.

By de Jong's theorem [1996, Theorem 4.1], there is an alteration $\phi: X^{\prime} \longrightarrow X$ and an open immersion $X^{\prime} \hookrightarrow \widehat{X}^{\prime}$ into a smooth projective integral scheme $\widehat{X}^{\prime}$. There is a nonempty open subscheme $U$ of $X$ such that the projection

$$
U^{\prime}:=U \times_{X} X^{\prime} \stackrel{f}{\longrightarrow} U
$$

is finite and étale of degree $\delta=\left[k\left(U^{\prime}\right): k(U)\right]$. The composition

$$
H_{c}^{2 d_{0}-i}\left(U, \mathbb{Q}\left(d_{0}-j\right)\right) \stackrel{f^{*}}{\longrightarrow} H_{c}^{2 d_{0}-i}\left(U^{\prime}, \mathbb{Q}\left(d_{0}-j\right)\right) \stackrel{f_{*}}{\longrightarrow} H_{c}^{2 d_{0}-i}\left(U, \mathbb{Q}\left(d_{0}-j\right)\right)
$$

is multiplication by $\delta(\neq 0)$, so it is an isomorphism. In particular, $f^{*}$ is injective. On the other hand, $H_{c}^{2 d_{0}-i}\left(U^{\prime}, \mathbb{Q}\left(d_{0}-j\right)\right)=0$ because $U^{\prime}$ is an open subscheme of a smooth projective integral scheme $\widehat{X}^{\prime}$ and

$$
H_{c}^{2 d_{0}-i}\left(\widehat{X}^{\prime}, \mathbb{Q}\left(d_{0}-j\right)\right) \cong H^{2 d_{0}-i}\left(\widehat{X}^{\prime}, \mathbb{Q}\left(d_{0}-j\right)\right) \cong H_{2 n-2 d_{0}+i}^{\mathrm{BM}}\left(\widehat{X}^{\prime}, \mathbb{Q}\left(n-d_{0}+j\right)\right)=0
$$

by Proposition 2.7 (we used $i, j \leq 0$ and $i-j \leq 2$ here). Hence, by the injectivity of $f^{*}$, we conclude that $H_{c}^{2 d_{0}-i}\left(U, \mathbb{Q}\left(d_{0}-j\right)\right)=0$. The lemma is proved.

Proof of Theorem 3.1. We may assume that $X$ is an integral scheme. Let us write $d:=\operatorname{dim} X$. If $U$ is an open subscheme of $X$, the associated localization sequence for motivic cohomology with compact supports [Kelly 2012, Proposition 5.5.5] gives an exact sequence

$$
\begin{aligned}
H_{c}^{2 d-i-1}(X \backslash U, \mathbb{Q}(d-j)) \longrightarrow & H_{i}(U, \mathbb{Q}(j)) \\
& \longrightarrow H_{i}(X, \mathbb{Q}(j)) \longrightarrow H_{c}^{2 d-i}(X \backslash U, \mathbb{Q}(d-j)) .
\end{aligned}
$$

Here, we used [Kelly 2012, Theorem 5.5.14(3)]. By Lemma 3.2, the first and the last terms vanish, so we have an isomorphism

$$
H_{i}(U, \mathbb{Q}(j)) \stackrel{\sim}{\rightarrow} H_{i}(X, \mathbb{Q}(j)) .
$$

As before, by de Jong's theorem, there is an alteration $\phi: X^{\prime} \longrightarrow X$ and a nonempty open immersion $X^{\prime} \hookrightarrow \widehat{X}^{\prime}$ into a smooth projective integral scheme $\widehat{X}^{\prime}$. There is an open subscheme $U$ of $X$ such that the projection

$$
U^{\prime}:=U \times_{X} X^{\prime} \stackrel{f}{\longrightarrow} U
$$


is a finite étale morphism of degree $\delta$. The composition

$$
H_{i}(U, \mathbb{Q}(j)) \stackrel{f^{*}}{\longrightarrow} H_{i}\left(U^{\prime}, \mathbb{Q}(j)\right) \stackrel{f_{*}}{\longrightarrow} H_{i}(U, \mathbb{Q}(j))
$$

is multiplication by $\delta$, so it is an isomorphism. In particular, $f^{*}$ is injective. But $H_{i}\left(U^{\prime}, \mathbb{Q}(j)\right)=0$ because $U^{\prime}$ is an open subscheme of a smooth projective integral scheme $\widehat{X}^{\prime}$, for which we know $H_{i}\left(\widehat{X}^{\prime}, \mathbb{Q}(j)\right)=0$ by Theorem 1.4 and Lemma 2.1 . Hence, we obtain $H_{i}(X, \mathbb{Q}(j)) \cong H_{i}(U, \mathbb{Q}(j))=0$. This proves the theorem.

\section{Proof of Theorem 1.1}

In the rest of this paper, we assume the existence of resolution of singularities in the sense of [Voevodsky et al. 2000, Chapter 4, Definition 3.4]. This assumption is needed even to deal with smooth schemes because our argument depends on the existence of smooth compactification. Alternatively, the reader may choose to assume that schemes have dimension at most 3.

Our proof of Theorem 1.1 goes as follows. We first prove the vanishing statement for $i \leq-2$. For smooth schemes, this is done by showing that a smooth compactification induces an isomorphism of motivic homology groups of certain indices (Proposition 4.2) and applying Kondo and Yasuda's result (Proposition 2.7). For a singular scheme, the statement is proved by induction on dimension using the abstract blowup sequence associated with a desingularization of the scheme (Proposition 4.3). In order to prove the statement for $i=-1$, we first deal with the smooth case by taking smooth compactification (Proposition 4.7). Then, combining these results, we construct the inverse to the canonical map $\alpha_{X}: H_{-1}(X, \mathbb{Z}(j)) \rightarrow$ $H_{-1}\left(\pi_{0}(X), \mathbb{Z}(j)\right)$ by using the universal property of a certain pushout diagram of motivic homology groups. We show that this diagram is indeed cocartesian by means of Galois cohomology, following the method of [Kondo and Yasuda 2013].

Lemma 4.1. Let $X$ be a scheme over $\mathbb{F}_{q}$ of dimension at most $d-1$. If $\operatorname{dim} X=0$,

$$
H^{2 d-i}(X, \mathbb{Z}(d-j))=0
$$

for $i, j \leq 0$. If $\operatorname{dim} X \geq 1$, a desingularization $\tilde{X} \longrightarrow X$ of $X$ induces isomorphisms

$$
H^{2 d-i}(X, \mathbb{Z}(d-j)) \stackrel{\sim}{\rightarrow} H^{2 d-i}(\tilde{X}, \mathbb{Z}(d-j))
$$

for $i, j \leq 1$ with $i-j \leq 2$.

Proof. If $\operatorname{dim} X=0, X_{\text {red }}$ is a finite disjoint union of spectra of finite fields over $\mathbb{F}_{q}$. Thus,

$$
H^{2 d-i}(X, \mathbb{Z}(d-j)) \cong H^{2 d-i}\left(X_{\text {red }}, \mathbb{Z}(d-j)\right) \cong H_{-2 d+i}^{\mathrm{BM}}\left(X_{\text {red }}, \mathbb{Z}(-d+j)\right)=0 .
$$


The first isomorphism follows because the motive $M(X)$ is isomorphic to the motive $M\left(X_{\text {red }}\right)$, and the second because $X_{\text {red }}$ is smooth. The last equality follows from Lemma 2.1 because $d \geq 1$ implies that $-2 d+i \leq-2$ and $-d+j \leq-1$.

We prove the second assertion by induction on the dimension of $X$. Let $Z$ be the closed subscheme of $X$ on which $\widetilde{X} \longrightarrow X$ is not an isomorphism. Then the abstract blowup

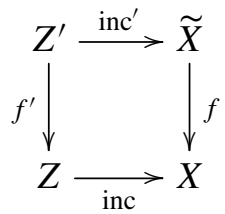

gives rise to a long exact sequence of motivic cohomology groups

$$
\begin{aligned}
\cdots \longrightarrow H^{2 d-i-1}\left(Z^{\prime}, \mathbb{Z}(d-j)\right) & \longrightarrow H^{2 d-i}(X, \mathbb{Z}(d-j)) \\
\stackrel{\left(f^{*}, \mathrm{inc}^{*}\right)}{\longrightarrow} & H^{2 d-i}(\tilde{X}, \mathbb{Z}(d-j)) \oplus H^{2 d-i}(Z, \mathbb{Z}(d-j)) \\
\stackrel{\mathrm{inc}^{\prime *}-f^{\prime *}}{\longrightarrow} & H^{2 d-i}\left(Z^{\prime}, \mathbb{Z}(d-j)\right) \longrightarrow \cdots .
\end{aligned}
$$

In order to show that $f^{*}$ is an isomorphism, we shall prove that the three cohomology groups of $Z$ and $Z^{\prime}$ vanish.

If $\operatorname{dim} X=1$, then $\operatorname{dim} Z=\operatorname{dim} Z^{\prime}=0$; so $Z_{\text {red }}$ and $Z_{\text {red }}^{\prime}$ are finite disjoint unions of spectra of finite fields over $\mathbb{F}_{q}$. Hence, it suffices to observe that the first claim of the lemma implies, for any finite field $\mathbb{F}$ over $\mathbb{F}_{q}$, the following (note that we are in the range $d-1 \geq 1$ ):

$$
\begin{aligned}
H^{2 d-i-1}(\operatorname{Spec} \mathbb{F}, \mathbb{Z}(d-j)) & =H^{2(d-1)-(i-1)}(\operatorname{Spec} \mathbb{F}, \mathbb{Z}((d-1)-(j-1)))=0, \\
H^{2 d-i}(\operatorname{Spec} \mathbb{F}, \mathbb{Z}(d-j)) & =H^{2(d-1)-(i-2)}(\operatorname{Spec} \mathbb{F}, \mathbb{Z}((d-1)-(j-1)))=0 .
\end{aligned}
$$

Now suppose that $\operatorname{dim} X \geq 2$ and assume that the lemma is known for schemes of smaller dimension. We shall again prove that the cohomology groups of $Z$ and $Z^{\prime}$ in the above long exact sequence vanish. Let us prove that $H^{2 d-i}(Z, \mathbb{Z}(d-j))=0$. Since $\operatorname{dim} Z<\operatorname{dim} X$, by the induction hypothesis, we have an isomorphism

$$
H^{2 d-i}(Z, \mathbb{Z}(d-j)) \stackrel{\sim}{\longrightarrow} H^{2 d-i}(\widetilde{Z}, \mathbb{Z}(d-j)),
$$

where $\widetilde{Z}$ is a desingularization of $Z$. Since $\widetilde{Z}$ is a smooth scheme, every connected component $Z_{r}\left(r=1, \cdots, r_{0}\right)$ of $\widetilde{Z}$ is also smooth. By [Mazza et al. 2006, Theorem 19.1],

$$
H^{2 d-i}(\widetilde{Z}, \mathbb{Z}(d-j)) \cong \bigoplus_{r=1}^{r_{0}} H^{2 d-i}\left(Z_{r}, \mathbb{Z}(d-j)\right)
$$




$$
\cong \bigoplus_{r=1}^{r_{0}} H_{2 \operatorname{dim} Z_{r}-2 d+i}^{\mathrm{BM}}\left(Z_{r}, \mathbb{Z}\left(\operatorname{dim} Z_{r}-d+j\right)\right) .
$$

The last group vanishes if $i \leq 2, j \leq 1$ and $i-j \leq 3$. Indeed, since $\operatorname{dim} Z_{r} \leq d-2$, when $i$ and $j$ satisfy these inequalities, we have

$$
\begin{array}{r}
2 \operatorname{dim} Z_{r}-2 d+i \leq 2(d-2)-2 d+i=i-4 \leq-2, \\
\operatorname{dim} Z_{r}-d+j \leq(d-2)-d+j=j-2 \leq-1,
\end{array}
$$

and

$$
\begin{aligned}
\left(2 \operatorname{dim} Z_{r}-2 d+i\right)-\left(\operatorname{dim} Z_{r}-d+j\right) & =\operatorname{dim} Z_{r}-d+i-j \\
& \leq i-j-2 \leq 1 .
\end{aligned}
$$

Hence, by Proposition 2.7, we obtain

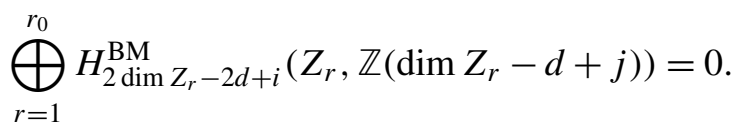

Similarly, we can calculate $H^{2 d-i}\left(Z^{\prime}, \mathbb{Z}(d-j)\right)=0$ if $i \leq 2, j \leq 1$ and $i-j \leq 3$, and $H^{2 d-i-1}\left(Z^{\prime}, \mathbb{Z}(d-j)\right)=0$ if $i \leq 1, j \leq 1$ and $i-j \leq 2$. Therefore, the lemma follows.

Proposition 4.2. Suppose $X$ is a connected smooth scheme of dimension d over $\mathbb{F}_{q}$. Then a smooth compactification $X \hookrightarrow X^{\prime}$ of $X$ induces isomorphisms

$$
H_{i}(X, \mathbb{Z}(j)) \stackrel{\sim}{\longrightarrow} H_{i}\left(X^{\prime}, \mathbb{Z}(j)\right)
$$

for all $i \leq-1$ and $j \leq 0$ with $i-j \leq 1$.

Proof. There is nothing to prove if $d=0$, so we deal with the case where $d \geq 1$. Since there is a localization sequence

$$
\begin{aligned}
H_{c}^{2 d-i-1}\left(X^{\prime} \backslash X, \mathbb{Z}(d-j)\right) \longrightarrow & H_{i}(X, \mathbb{Z}(j)) \\
& \longrightarrow H_{i}\left(X^{\prime}, \mathbb{Z}(j)\right) \longrightarrow H_{c}^{2 d-i}\left(X^{\prime} \backslash X, \mathbb{Z}(d-j)\right)
\end{aligned}
$$

and $X^{\prime} \backslash X$ is proper, it suffices to show $H^{2 d-i-1}\left(X^{\prime} \backslash X, \mathbb{Z}(d-j)\right)=0$ and $H^{2 d-i}\left(X^{\prime} \backslash X, \mathbb{Z}(d-j)\right)=0$. We prove this for the first group. The proof for the second group is identical. First, note that by the first assertion of Lemma 4.1, we may assume that the irreducible components of $X^{\prime} \backslash X$ have nonzero dimension. Now, let $\widetilde{X^{\prime} \backslash X}$ be a desingularization of $X^{\prime} \backslash X$ and write its decomposition into connected components as $\widetilde{X^{\prime} \backslash X}=\bigsqcup_{s=1}^{s_{0}} X_{s}$. With the second assertion of 
Lemma 4.1, we can calculate

$$
\begin{aligned}
H^{2 d-i-1}\left(X^{\prime} \backslash X, \mathbb{Z}(d-j)\right) & \cong H^{2 d-i-1}\left(\widetilde{X^{\prime} \backslash X}, \mathbb{Z}(d-j)\right) \\
& \cong \bigoplus_{s=1}^{s_{0}} H^{2 d-i-1}\left(X_{s}, \mathbb{Z}(d-j)\right) \\
& \cong \bigoplus_{s=1}^{s_{0}} H_{2 \operatorname{dim} X_{s}-2 d+i+1}^{\mathrm{BM}}\left(X_{s}, \mathbb{Z}\left(\operatorname{dim} X_{s}-d+j\right)\right),
\end{aligned}
$$

and the last group vanishes if $i \leq-1, j \leq 0$ and $i-j \leq 1$ by Proposition 2.7.

We are now able to prove the first half of Theorem 1.1.

Proposition 4.3. Let $X$ be a scheme over $\mathbb{F}_{q}$. Then $H_{i}(X, \mathbb{Z}(j))=0$ if $i \leq-2$, $j \leq-1$ and $i-j \leq 1$.

Proof. If $\operatorname{dim} X=0$, the proposition holds by Lemma 2.1. Let us assume that $\operatorname{dim} X \geq 1$ and prove the proposition by induction on $\operatorname{dim} X$. Let $Z$ be a closed subscheme of $X$ which contains all singular points of $X$ and has dimension less than that of $X$. The abstract blowup

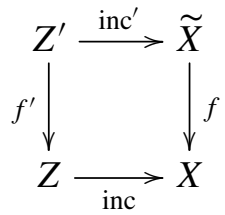

gives rise to a long exact sequence

$$
\begin{aligned}
H_{i}\left(Z^{\prime}, \mathbb{Z}(j)\right) \stackrel{\left(f_{*}^{\prime}, \mathrm{inc}_{*}^{\prime}\right)}{\longrightarrow} & H_{i}(Z, \mathbb{Z}(j)) \oplus H_{i}(\tilde{X}, \mathbb{Z}(j)) \\
& \stackrel{\mathrm{inc}_{*}-f_{*}}{\longrightarrow} H_{i}(X, \mathbb{Z}(j)) \longrightarrow H_{i-1}\left(Z^{\prime}, \mathbb{Z}(j)\right) .
\end{aligned}
$$

By the induction hypothesis,

$$
H_{i}\left(Z^{\prime}, \mathbb{Z}(j)\right)=0, \quad H_{i}(Z, \mathbb{Z}(j))=0 \quad \text { and } \quad H_{i-1}\left(Z^{\prime}, \mathbb{Z}(j)\right)=0 .
$$

Hence,

$$
H_{i}(X, \mathbb{Z}(j)) \cong H_{i}(\tilde{X}, \mathbb{Z}(j)) \cong H_{i}\left(\tilde{X}^{\prime}, \mathbb{Z}(j)\right)=0,
$$

where $\tilde{X}^{\prime}$ denotes a smooth compactification of $\tilde{X}$, the second isomorphism follows from Proposition 4.2, and the last group vanishes by Proposition 2.7.

Next we shall consider the case where $i=-1$.

Lemma 4.4. Let $X$ be a geometrically connected scheme over a field $k$ and $i$ : $X \hookrightarrow X^{\prime}$ be a compactification, i.e., an open immersion into a proper scheme $X^{\prime}$ with dense image. Then $X^{\prime}$ is geometrically connected over $k$. 
Proof. Since $X^{\prime}$ is connected, it is enough to show that $\pi_{0}\left(X^{\prime}\right)$ has a $k$-rational point [Liu 2002, Chapter 10, Corollary 2.21(a)]. Now, $i$ induces a $k$-morphism $\pi_{0}(X) \longrightarrow \pi_{0}\left(X^{\prime}\right)$. Since $X$ is geometrically connected over a field, $\pi_{0}(X)=$ Spec $k$. So this morphism defines a $k$-rational point on $\pi_{0}\left(X^{\prime}\right)$.

Remark 4.5. With the same notation, the above proof shows that $\pi_{0}(X)=\pi_{0}\left(X^{\prime}\right)$.

We need the independence of motivic homology from the choice of the base field.

Lemma 4.6. If $l / k$ is a finite extension of fields and $X$ is a scheme of finite type over $l$, we have a canonical isomorphism

$$
\operatorname{Hom}_{\mathrm{DM}_{\mathrm{Nis}}^{-}(k)}(\mathbb{Z}(j)[i], M(X)) \cong \operatorname{Hom}_{\mathrm{DM}_{\mathrm{Nis}}^{-}(l)}(\mathbb{Z}(j)[i], M(X))
$$

for all $i \in \mathbb{Z}$ and $j \in \mathbb{Z}_{\leq 0}$, where, on the left-hand side, $X$ is regarded as a scheme over $k$ by the composition $X \stackrel{\text { str }}{\longrightarrow} \operatorname{Spec} l \rightarrow \operatorname{Spec} k$.

Proof. For $j<0$, by [Mazza et al. 2006, Corollary 15.3] and [Voevodsky 2010, Corollary 4.10], there is an isomorphism

$$
\operatorname{Hom}_{\mathrm{DM}_{\mathrm{Nis}}^{-}(l)}(\mathbb{Z}(j)[i], M(X)) \cong H_{i-2 j-1}\left(\frac{\operatorname{Cor}_{l}\left(\Delta_{l}^{*}, X \times_{l}\left(\mathbb{A}_{l}^{-j}-\{0\}\right)\right)}{\operatorname{Cor}_{l}\left(\Delta_{l}^{*}, X \times_{l}\{1\}\right)}\right),
$$

where Cor denotes the group of finite correspondences. Since $l$ is a finite extension of $k$, if $S$ is a scheme over $l$ and $T$ is over $k$, we have $\operatorname{Cor}_{l}\left(T \times_{k} l, S\right) \cong \operatorname{Cor}_{k}(T, S)$. Hence, the right-hand side is isomorphic to

$$
H_{i-2 j-1}\left(\frac{\operatorname{Cor}_{k}\left(\Delta_{k}^{*}, X \times_{k}\left(\mathbb{A}_{k}^{-j}-\{0\}\right)\right)}{\operatorname{Cor}_{k}\left(\Delta_{k}^{*}, X \times_{k}\{1\}\right)}\right),
$$

which is, in turn, isomorphic to $\operatorname{Hom}_{\mathrm{DM}_{\mathrm{Nis}}^{-}(k)}(\mathbb{Z}(j)[i], M(X))$.

Proposition 4.7. If $X$ is smooth over $\mathbb{F}_{q}$, there are canonical isomorphisms

$$
\phi: H_{-1}(X, \mathbb{Z}(j)) \stackrel{\sim}{\longrightarrow} H_{-1}\left(\pi_{0}(X), \mathbb{Z}(j)\right)
$$

for $j=-1$ and -2 .

Proof. We may assume that $X$ is connected and regard it as a scheme over $\pi_{0}(X)$ by Lemma 4.6. Now $X$ is geometrically connected as a scheme over $\pi_{0}(X)$, so its smooth compactification $X^{\prime}$ is also smooth over $\pi_{0}(X)$ by Lemma 4.4. Now, the map $\phi$ fits in the commutative diagram

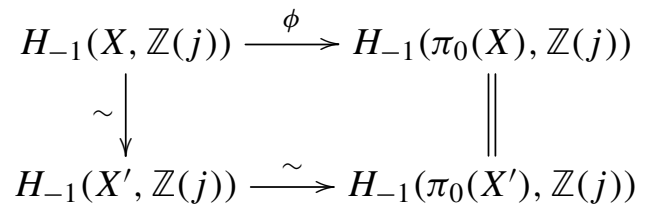


where the left vertical map is an isomorphism by Proposition 4.2, the bottom horizontal map is an isomorphism by Theorem 1.4 and the right vertical equality follows from Remark 4.5. Thus, $\phi$ is an isomorphism.

In order to compute motivic homology of singular schemes, we shall now study how motivic homology groups behave under resolution of singularities.

Lemma 4.8 [Kondo and Yasuda 2013, Lemma 2.7]. For two finite fields $\mathbb{F}_{q^{n}} \subset \mathbb{F}_{q^{m}}$, the canonical map

$$
H_{-1}\left(\operatorname{Spec} \mathbb{F}_{q^{m}}, \mathbb{Z}(j)\right) \longrightarrow H_{-1}\left(\operatorname{Spec} \mathbb{F}_{q^{n}}, \mathbb{Z}(j)\right)
$$

is surjective if $j<0$.

Proof. As we have seen in the proof of Lemma 2.1, the cycle class map gives an isomorphism

$$
H_{-1}(\operatorname{Spec} \mathbb{F}, \mathbb{Z}(j)) \cong \bigoplus_{l \neq p} H_{\mathrm{ett}}^{0}\left(\operatorname{Spec} \mathbb{F}, \mathbb{Q}_{l} / \mathbb{Z}_{l}(-j)\right)
$$

for $j \leq-1$ and a finite field $\mathbb{F}$. Now, the cycle class map is compatible with the pushforward along a finite morphism [Geisser and Levine 2001, Lemma 3.5(2)], so the surjectivity follows from the corresponding statement for étale cohomology [Soulé 1979, Lemma 6(iii), p. 269 and IV.1.7, p. 283].

Lemma 4.9. Let $X$ be a scheme over $\mathbb{F}_{q}, f: \tilde{X} \longrightarrow X$ be a desingularization, and $j=-1$ or -2 . Then the map

$$
f_{*}: H_{-1}(\tilde{X}, \mathbb{Z}(j)) \longrightarrow H_{-1}(X, \mathbb{Z}(j))
$$

is surjective.

Proof. We prove this by induction on the dimension of $X$. Let $Z$ be a closed subscheme of $X$ which contains all singularities of $X$ and has dimension less than that of $X$. The abstract blowup

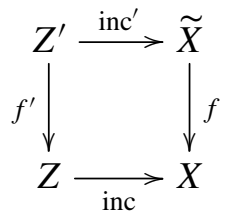

gives rise to a long exact sequence

$$
\begin{aligned}
H_{-1}\left(Z^{\prime}, \mathbb{Z}(j)\right) \stackrel{\left(f_{*}^{\prime}, \mathrm{inc}_{*}^{\prime}\right)}{\longrightarrow} H_{-1}(Z, \mathbb{Z}(j)) \oplus H_{-1}(\tilde{X}, \mathbb{Z}(j)) & \stackrel{\text { inc }_{*}-f_{*}}{\longrightarrow} H_{-1}(X, \mathbb{Z}(j)) \stackrel{\delta}{\longrightarrow} H_{-2}\left(Z^{\prime}, \mathbb{Z}(j)\right)=0,
\end{aligned}
$$


where $\delta$ is the connecting map. The last term vanishes by Proposition 4.3. By an easy diagram chase, in order to show the surjectivity of $f_{*}$, it is enough to show the surjectivity of $f_{*}^{\prime}$. Let us write $Z=\bigcup Z_{i}$, where $Z_{i}$ are the irreducible components of $Z$, and let $\widetilde{Z}_{i}$ be a desingularization of $Z_{i}$ and $p: \bigsqcup \widetilde{Z}_{i} \longrightarrow \bigcup Z_{i}$ be the morphism induced by the desingularizations. Note that $p$ is then a desingularization of $Z$. For each index $i$, choose a closed point $x_{i} \in \widetilde{Z}_{i}$. Let $y_{i}:=p\left(x_{i}\right) \in Z_{i} \subset Z$ be the image of $x_{i}$ under $p$. Since $f^{\prime}$ is surjective, there is a closed point $z_{i} \in Z^{\prime}$ with $f^{\prime}\left(z_{i}\right)=y_{i}$ for each $i$. Choose some finite field extension $\mathbb{F}$ of $\mathbb{F}_{q}$ which contains all residue fields $k\left(x_{i}\right), k\left(y_{i}\right)$ and $k\left(z_{i}\right)$. The inclusions of these residue fields into $\mathbb{F}$ give rise to $\mathbb{F}$-rational points

$$
\begin{aligned}
& x_{i}: \operatorname{Spec} \mathbb{F} \longrightarrow \operatorname{Spec} k\left(x_{i}\right) \longrightarrow X, \\
& y_{i}: \operatorname{Spec} \mathbb{F} \longrightarrow \operatorname{Spec} k\left(y_{i}\right) \longrightarrow X, \\
& z_{i}: \operatorname{Spec} \mathbb{F} \longrightarrow \operatorname{Spec} k\left(z_{i}\right) \longrightarrow X,
\end{aligned}
$$

which are, with an abuse of notation, denoted by the same letters $x_{i}, y_{i}$ and $z_{i}$. These points give the commutative diagram

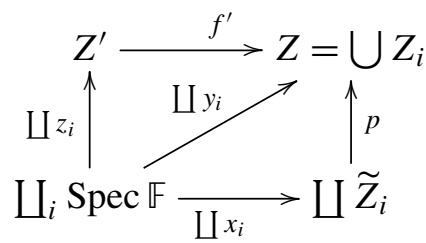

Taking homology groups, we obtain

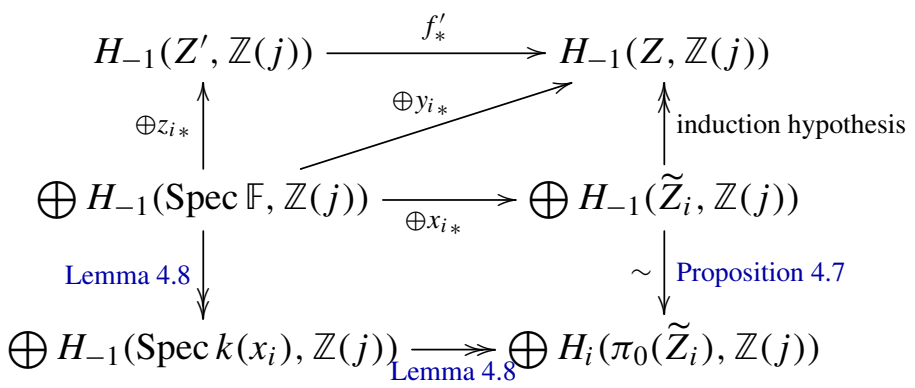

Hence $f_{*}^{\prime}$ is surjective.

The next lemma compares the motivic homology of a given scheme with the motivic homology of one of its irreducible components.

Lemma 4.10. Let $X$ be a connected scheme over $\mathbb{F}_{q}$ and $X_{1}$ be an irreducible component. If $j=-1$ or -2 , the inclusion of $X_{1}$ into $X$ induces a surjection

$$
H_{-1}\left(X_{1}, \mathbb{Z}(j)\right) \longrightarrow H_{-1}(X, \mathbb{Z}(j)) .
$$


Proof. Let us write $X=X_{1} \cup X_{2} \cup \cdots \cup X_{r}$, where the $X_{i}$ are the irreducible components of $X$. Since the lemma is obvious for $r=1$, we assume that $r>1$ below. The abstract blowup

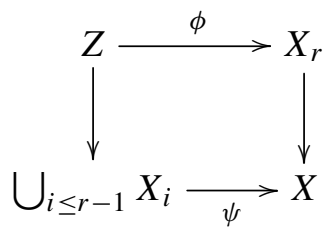

(all the maps in the diagram are inclusions) gives an exact sequence

$$
\begin{aligned}
H_{-1}(Z, \mathbb{Z}(j)) \longrightarrow H_{-1}\left(X_{r}, \mathbb{Z}(j)\right) \oplus H_{-1}\left(\bigcup_{i \leq r-1} X_{i}, \mathbb{Z}(j)\right) \\
\longrightarrow H_{-1}(X, \mathbb{Z}(j)) \longrightarrow H_{-2}(Z, \mathbb{Z}(j))=0,
\end{aligned}
$$

where the last equality comes from Proposition 4.3. By induction on the number of irreducible components of $X$, it suffices to prove the surjectivity of $\psi_{*}$ : $H_{-1}\left(\bigcup_{i<r-1} X_{i}, \mathbb{Z}(j)\right) \longrightarrow H_{-1}(X, \mathbb{Z}(j))$, which, in turn, follows from the surjectivity of $\phi_{*}: H_{-1}(Z, \mathbb{Z}(j)) \longrightarrow H_{-1}\left(X_{r}, \mathbb{Z}(j)\right)$.

Since $X$ is connected, $Z$ is not empty. In particular, it has a closed point, say $z \in Z$. Choose a desingularization $\pi: \widetilde{X}_{r} \longrightarrow X_{r}$ and let a closed point $\widetilde{w} \in \widetilde{X}_{r}$ be a preimage of $w:=\phi(z) \in X_{r}$. Choose some finite field extension $\mathbb{F}$ of $\mathbb{F}_{q}$ containing all the residue fields $k(z), k(w)$ and $k(\widetilde{w})$, and regard $z, w$ and $\widetilde{w}$ as $\mathbb{F}$-rational points. Now, there is a commutative diagram

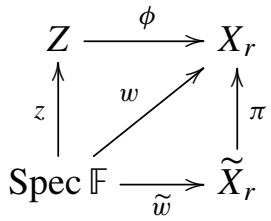

Passing to homology groups, we obtain (noting that $\mathcal{O}\left(\pi_{0}\left(\widetilde{X}_{r}\right)\right) \subset k(\widetilde{w}) \subset \mathbb{F}$ ) the commutative diagram

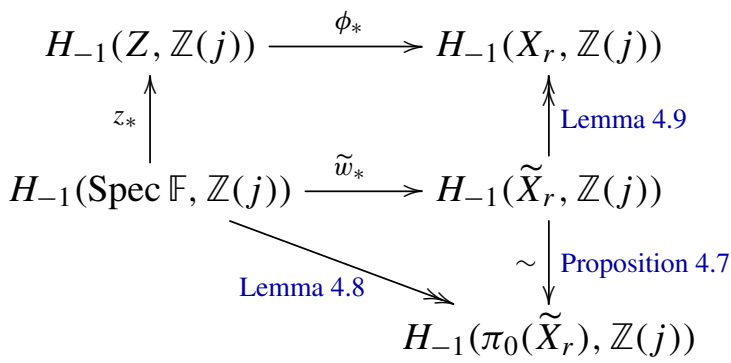

Hence, $\phi_{*}$ is surjective. 
Proof of Theorem 1.1. We have already proved the first half in Proposition 4.3. It remains to prove the second half, i.e., the following statement:

Let $X$ be an arbitrary scheme over $\mathbb{F}_{q}$ and $j=-1$ or -2 . Then the canonical map $\alpha_{X}: H_{-1}(X, \mathbb{Z}(j)) \longrightarrow H_{-1}\left(\pi_{0}(X), \mathbb{Z}(j)\right)$ is an isomorphism.

If $\operatorname{dim} X=0$, then $\alpha_{X}$ is clearly an isomorphism (because we may assume $X$ to be a disjoint union of reduced schemes, i.e., a union of spectra of finite fields).

We prove the theorem by induction on the dimension of $X$. Assume that the theorem holds for schemes of dimension at most $d-1$. We prove the assertion for a $d$-dimensional scheme $X$. By Lemma 4.6, we may assume without loss of generality that $X$ is geometrically connected and reduced. Choose a nonempty closed subscheme $Z$ of $X$ such that $X \backslash Z$ is smooth and $\operatorname{dim} Z<\operatorname{dim} X$. First, we claim that the inclusion $Z \hookrightarrow X$ induces a surjection

$$
\beta: H_{-1}(Z, \mathbb{Z}(j)) \longrightarrow H_{-1}(X, \mathbb{Z}(j)) .
$$

Indeed, there is some irreducible component, call it $X_{1}$, of $X$ such that $Z \cap X_{1} \neq \varnothing$. Let $\tilde{X}_{1}$ be a desingularization of $X_{1}$. Choose a closed point $x \in Z \cap X_{1}$ and its preimage $\tilde{x} \in \tilde{X}_{1}$. Let $\mathbb{F}$ be a sufficiently large finite field that contains both residue fields $k(x)$ and $k(\tilde{x})$. (Note that $k(\tilde{x})$ contains $\pi_{0}\left(\tilde{X}_{1}\right)$.) Then there is a commutative diagram

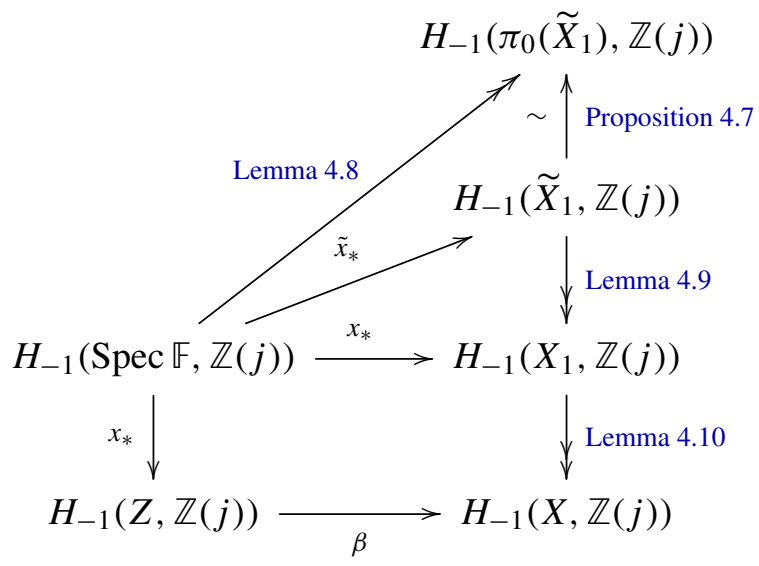

The commutativity of the diagram implies the surjectivity of $\beta$.

Next, consider the commutative diagram

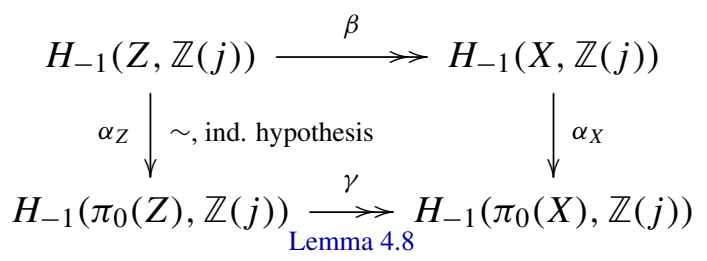


In order to show that $\alpha_{X}$ is an isomorphism, it is enough to show its injectivity, for the surjectivity is obvious from the diagram. The injectivity follows once one constructs a group homomorphism

$$
l: H_{-1}\left(\pi_{0}(X), \mathbb{Z}(j)\right) \longrightarrow H_{-1}(X, \mathbb{Z}(j))
$$

such that $l \circ \gamma \circ \alpha_{Z}=\beta$, because then the surjectivity of $\beta$ and the equalities $l \circ \alpha_{X} \circ \beta=l \circ \gamma \circ \alpha_{Z}=\beta$ imply that $l \circ \alpha_{X}=$ id. (In fact, $l$ is the inverse to $\alpha_{X}$ because we also have $\alpha_{X} \circ l \circ \gamma=\alpha_{X} \circ \beta \circ \alpha_{Z}^{-1}=\gamma$, and the surjectivity of $\gamma$ implies that $\alpha_{X} \circ l=$ id.)

The existence of such a map $l$ follows if one shows that the square in the following diagram is cocartesian:

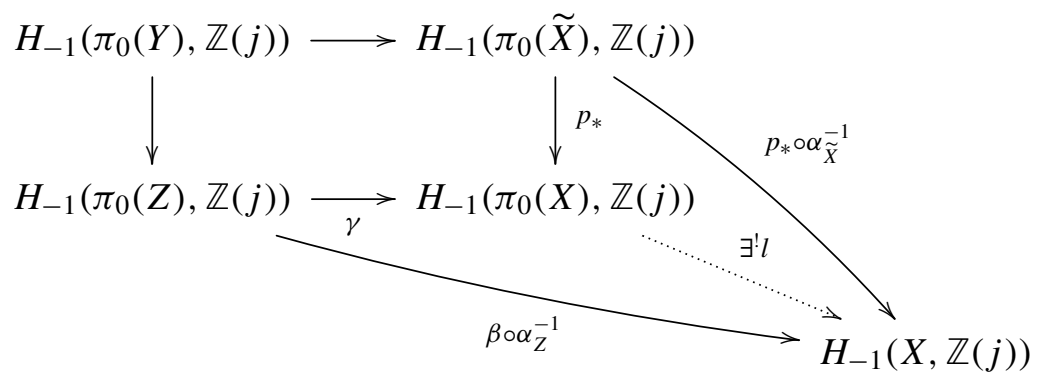

Here, $p: \widetilde{X} \longrightarrow X$ is a desingularization of $X, Y:=\left(\widetilde{X} \times_{X} Z\right)_{\text {red }}$ and $\gamma$ is the map induced by the canonical morphism $\pi_{0}(Z) \longrightarrow \pi_{0}(X)$. Indeed, the map $l$ defined by universality in the above diagram satisfies $l \circ \gamma \circ \alpha_{Z}=\beta$ by its definition. Note that $\alpha_{\tilde{X}}^{-1}$ makes sense because $\alpha_{\tilde{X}}$ is an isomorphism by Proposition 4.7 , and so does $\alpha_{Z}^{-1}$ by the induction hypothesis.

Since for a zero-dimensional $\mathbb{F}_{q}$-scheme $S$ and $j \leq-1$ there is an isomorphism

$$
H_{-1}(S, \mathbb{Z}(j)) \cong \bigoplus_{l \neq p} H_{\text {et }}^{0}\left(S, \mathbb{Q}_{l} / \mathbb{Z}_{l}(-j)\right)
$$

that is functorial with respect to pushforward along finite morphisms [Geisser and Levine 2001, Lemma 3.5(2)] induced by the Geisser-Levine cycle map, it is enough to show that the diagram

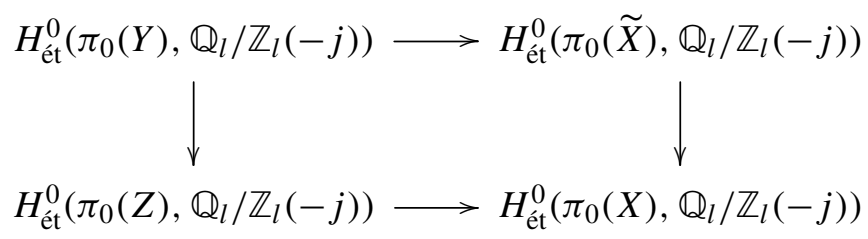

is cocartesian for all primes $l \neq p$. (Here, the arrows are pushforward maps along finite morphisms.) 
Now, consider the diagram

$$
\begin{gathered}
H_{\text {êt }}^{0}\left(\pi_{0}(\bar{Y}), \mathbb{Q}_{l} / \mathbb{Z}_{l}(-j)\right) \stackrel{a}{\longrightarrow} H_{\text {êt }}^{0}\left(\pi_{0}(\overline{\widetilde{X}}), \mathbb{Q}_{l} / \mathbb{Z}_{l}(-j)\right) \\
b \downarrow \\
\downarrow \\
H_{\text {êt }}^{0}\left(\pi_{0}(\bar{Z}), \mathbb{Q}_{l} / \mathbb{Z}_{l}(-j)\right) \underset{d}{\longrightarrow} H_{\text {êt }}^{0}\left(\pi_{0}(\bar{X}), \mathbb{Q}_{l} / \mathbb{Z}_{l}(-j)\right)
\end{gathered}
$$

where ${ }^{-}$indicates the base change to the algebraic closure $\overline{\mathbb{F}}_{q}$; for example, $\bar{X}=$ $X \otimes_{\mathbb{F}_{q}} \overline{\mathbb{F}}_{q}$.

Let us for the moment assume that the diagram $(* *)$ is cocartesian in the category of $G\left(\overline{\mathbb{F}}_{q} / \mathbb{F}_{q}\right)$-modules and that the module

$$
\begin{aligned}
N:=\operatorname{ker}\left\{H_{\mathrm{et}}^{0}\left(\pi_{0}(\bar{Y}), \mathbb{Q}_{l} / \mathbb{Z}_{l}(-j)\right)\right. & \\
& \left.\stackrel{(a, b)}{\longrightarrow} H_{\text {èt }}^{0}\left(\pi_{0}(\overline{\widetilde{X}}), \mathbb{Q}_{l} / \mathbb{Z}_{l}(-j)\right) \oplus H_{\text {êt }}^{0}\left(\pi_{0}(\bar{Z}), \mathbb{Q}_{l} / \mathbb{Z}_{l}(-j)\right)\right\}
\end{aligned}
$$

is divisible. We shall show that the diagram $(*)$ is cocartesian under these assumptions. Since the diagram $(* *)$ is a pushout, there is an exact sequence of $G\left(\overline{\mathbb{F}}_{q} / \mathbb{F}_{q}\right)$-modules

$0 \longrightarrow H_{\mathrm{et}}^{0}\left(\pi_{0}(\bar{Y}), \mathbb{Q}_{l} / \mathbb{Z}_{l}(-j)\right) / N$

$$
\begin{aligned}
\stackrel{(a, b)}{\longrightarrow} H_{\mathrm{et}}^{0}\left(\pi_{0}(\overline{\widetilde{X}}), \mathbb{Q}_{l} / \mathbb{Z}_{l}(-j)\right) \oplus & H_{\mathrm{et}}^{0}\left(\pi_{0}(\bar{Z}), \mathbb{Q}_{l} / \mathbb{Z}_{l}(-j)\right) \\
& \stackrel{c-d}{\longrightarrow} H_{\mathrm{et}}^{0}\left(\pi_{0}(\bar{X}), \mathbb{Q}_{l} / \mathbb{Z}_{l}(-j)\right) \longrightarrow 0,
\end{aligned}
$$

where $(a, b)$ is, of course, the quotient map induced by the map $(a, b)$ defined on $H_{\text {ét }}^{0}\left(\pi_{0}(\bar{Y}), \mathbb{Q}_{l} / \mathbb{Z}_{l}(-j)\right)$. Taking Galois cohomology of $G\left(\overline{\mathbb{F}}_{q} / \mathbb{F}_{q}\right)$-modules, we obtain the long exact sequence

$$
\begin{aligned}
& 0 \rightarrow\left(H_{\text {êt }}^{0}\left(\pi_{0}(\bar{Y}), \mathbb{Q}_{l} / \mathbb{Z}_{l}(-j)\right) / N\right)^{G\left(\mathbb{F}_{q} / \mathbb{F}_{q}\right)} \\
& \rightarrow H_{\text {êt }}^{0}\left(\pi_{0}(\tilde{X}), \mathbb{Q}_{l} / \mathbb{Z}_{l}(-j)\right) \oplus H_{\text {êt }}^{0}\left(\pi_{0}(Z), \mathbb{Q}_{l} / \mathbb{Z}_{l}(-j)\right) \rightarrow H_{\text {êt }}^{0}\left(\pi_{0}(X), \mathbb{Q}_{l} / \mathbb{Z}_{l}(-j)\right) \\
& \rightarrow H^{1}\left(G\left(\overline{\mathbb{F}}_{q} / \mathbb{F}_{q}\right), H_{\text {êt }}^{0}\left(\pi_{0}(\bar{Y}), \mathbb{Q}_{l} / \mathbb{Z}_{l}(-j)\right) / N\right) \rightarrow \cdots .
\end{aligned}
$$

Since $j \neq 0$, the Frobenius automorphism acts nontrivially on the divisible group $H_{\mathrm{ett}}^{0}\left(\pi_{0}(\bar{Y}), \mathbb{Q}_{l} / \mathbb{Z}_{l}(-j)\right) / N$, which is just a direct sum of copies of the divisible group $\mathbb{Q}_{l} / \mathbb{Z}_{l}(-j) / N$. By the same reasoning as in the last part of the proof of Lemma 2.1, we conclude that

$$
H^{1}\left(G\left(\overline{\mathbb{F}}_{q} / \mathbb{F}_{q}\right), H_{\text {ét }}^{0}\left(\pi_{0}(\bar{Y}), \mathbb{Q}_{l} / \mathbb{Z}_{l}(-j)\right) / N\right)=0 .
$$

Similarly, the short exact sequence

$$
0 \longrightarrow N \longrightarrow H_{\mathrm{ett}}^{0}\left(\pi_{0}(\bar{Y}), \mathbb{Q}_{l} / \mathbb{Z}_{l}(-j)\right) \longrightarrow H_{\mathrm{et}}^{0}\left(\pi_{0}(\bar{Y}), \mathbb{Q}_{l} / \mathbb{Z}_{l}(-j)\right) / N \longrightarrow 0
$$


gives rise to a long exact sequence in Galois cohomology

$0 \longrightarrow N^{G\left(\overline{\mathbb{F}}_{q} / \mathbb{F}_{q}\right)} \longrightarrow H_{\mathrm{ett}}^{0}\left(\pi_{0}(Y), \mathbb{Q}_{l} / \mathbb{Z}_{l}(-j)\right)$

$$
\longrightarrow\left(H_{\mathrm{et}}^{0}\left(\pi_{0}(\bar{Y}), \mathbb{Q}_{l} / \mathbb{Z}_{l}(-j)\right) / N\right)^{G\left(\overline{\mathbb{F}}_{q} / \mathbb{F}_{q}\right)} \longrightarrow H^{1}\left(G\left(\overline{\mathbb{F}}_{q} / \mathbb{F}_{q}\right), N\right)=0 .
$$

The last term vanishes because $N$ is assumed divisible and the Galois action is nontrivial if $N \neq 0$. (Since $N$ must have infinite cardinality, a trivial Galois action would imply that $N^{\prime}=N^{G\left(\overline{\mathbb{F}}_{q} / \mathbb{F}_{q}\right)}$ is infinite, but this would contradict the fact that $N^{\prime}$ is a subgroup of the finite group $H_{\text {ét }}^{0}\left(\pi_{0}(\bar{Y}), \mathbb{Q}_{l} / \mathbb{Z}_{l}(-j)\right)^{G\left(\overline{\mathbb{F}}_{q} / \mathbb{F}_{q}\right)}$.) In particular, the map

$$
H_{\text {ét }}^{0}\left(\pi_{0}(Y), \mathbb{Q}_{l} / \mathbb{Z}_{l}(-j)\right) \longrightarrow\left(H_{\text {êt }}^{0}\left(\pi_{0}(\bar{Y}), \mathbb{Q}_{l} / \mathbb{Z}_{l}(-j)\right) / N\right)^{G\left(\overline{\mathbb{F}}_{q} / \mathbb{F}_{q}\right)}
$$

is surjective.

Combining all these, we obtain an exact sequence

$$
\begin{array}{r}
H_{\mathrm{êt}}^{0}\left(\pi_{0}(Y), \mathbb{Q}_{l} / \mathbb{Z}_{l}(-j)\right) \longrightarrow H_{\mathrm{èt}}^{0}\left(\pi_{0}(\tilde{X}), \mathbb{Q}_{l} / \mathbb{Z}_{l}(-j)\right) \oplus H_{\mathrm{ét}}^{0}\left(\pi_{0}(Z), \mathbb{Q}_{l} / \mathbb{Z}_{l}(-j)\right) \\
\longrightarrow H_{\mathrm{èt}}^{0}\left(\pi_{0}(X), \mathbb{Q}_{l} / \mathbb{Z}_{l}(-j)\right) \longrightarrow 0 .
\end{array}
$$

This means that the diagram $(*)$ is cocartesian.

It now remains to prove that the diagram $(* *)$ is cocartesian and $N$ is a divisible group. Using the Pontryagin duality

$$
H_{\text {êt }}^{0}\left(T, \mathbb{Z}_{l}(j)\right) \cong \operatorname{Hom}_{\mathbb{Z}}\left(H_{\text {êt }}^{0}\left(T, \mathbb{Q}_{l} / \mathbb{Z}_{l}(-j)\right), \mathbb{Q} / \mathbb{Z}\right)
$$

for a zero-dimensional scheme $T$ over $\overline{\mathbb{F}}_{q}$, obtained by taking the inverse limit over $r$ of the duality

$$
H_{\text {êt }}^{0}\left(T, \mathbb{Z} / l^{r}(j)\right) \cong \operatorname{Hom}_{\mathbb{Z}}\left(H_{\text {êt }}^{0}\left(T, \mathbb{Z} / l^{r}(-j)\right), \mathbb{Q} / \mathbb{Z}\right),
$$

we can see that it suffices to prove that the diagram with pullback homomorphisms

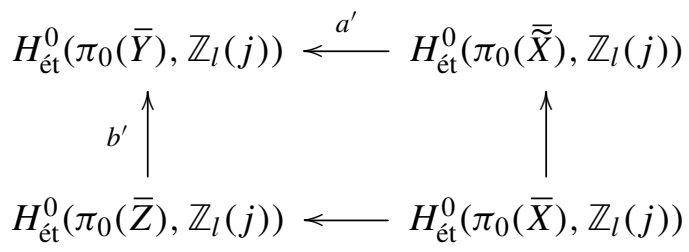

is cartesian and the cokernel of

$$
H_{\text {êt }}^{0}\left(\pi_{0}(\overline{\widetilde{X}}), \mathbb{Z}_{l}(j)\right) \oplus H_{\mathrm{ett}}^{0}\left(\pi_{0}(\bar{Z}), \mathbb{Z}_{l}(j)\right) \stackrel{a^{\prime}+b^{\prime}}{\longrightarrow} H_{\mathrm{e} t}^{0}\left(\pi_{0}(\bar{Y}), \mathbb{Z}_{l}(j)\right)
$$

is torsion-free. But, since there are canonical isomorphisms

$$
H_{\mathrm{et}}^{0}\left(\bar{X}, \mathbb{Z}_{l}(j)\right) \cong \operatorname{Hom}_{\mathrm{Set}}\left(\pi_{0}(\bar{X}), \mathbb{Z}_{l}\right) \otimes_{\mathbb{Z}_{l}} \mathbb{Z}_{l}(j)
$$


of $G\left(\overline{\mathbb{F}}_{q} / \mathbb{F}_{q}\right)$-modules (observe that these groups are just direct sums of $\mathbb{Z}_{l}(j)$ with one summand for each connected component of $\bar{X}$ ), it boils down to showing that the diagram

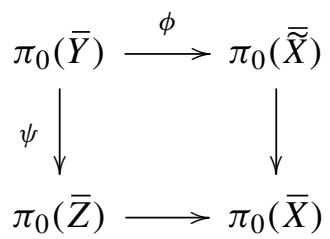

where $\phi$ and $\psi$ are the canonical maps, is cocartesian in the category of sets and the cokernel of the map

$$
\operatorname{Hom}_{\text {Set }}\left(\pi_{0}(\overline{\widetilde{X}}), \mathbb{Z}_{l}\right) \oplus \operatorname{Hom}_{\mathrm{Set}}\left(\pi_{0}(\bar{Z}), \mathbb{Z}_{l}\right) \longrightarrow \operatorname{Hom}_{\mathrm{Set}}\left(\pi_{0}(\bar{Y}), \mathbb{Z}_{l}\right)
$$

sending $(f, g)$ to $f \circ \phi+g \circ \psi$ is torsion-free. The claim about the cokernel is straightforward. (The proof can be found in [Kondo and Yasuda 2013, Lemma 3.3].)

Let us prove the assertion on the square diagram. Because the map $\psi$ is surjective and $\pi_{0}(\bar{X})$ consists of one element as we are working with a geometrically connected scheme $X$, it suffices to show that any two elements $x_{1}$ and $x_{2}$ in $\pi_{0}(\overline{\widetilde{X}})$ are related by the equivalence relation generated by the relation $\sim$ on $\pi_{0}(\widetilde{X})$ defined by $s \sim s^{\prime}$ if there are $t, t^{\prime} \in \pi_{0}(\bar{Y})$ such that $\phi(t)=s, \phi\left(t^{\prime}\right)=s^{\prime}$ and $\psi(t)=\psi\left(t^{\prime}\right)$. In order to prove this, we may assume that $x_{1}$ and $x_{2}$ in $\pi_{0}(\overline{\widetilde{X}})$ correspond to irreducible components $C_{1}$ and $C_{2}$ of $\bar{X}$ with nonempty intersection $C_{1} \cap C_{2}$. (If $x_{1}$ and $x_{2}$ correspond to irreducible elements $C_{1}$ and $C_{2}$ with empty intersection, choose a sequence of elements $x_{1}=s_{1}, s_{2}, \ldots, s_{r-1}, s_{r}=x_{2} \in \pi_{0}(\overline{\widetilde{X}})$ such that their corresponding irreducible components $C_{1}=S_{1}, S_{2}, \ldots, S_{r-1}, S_{r}=C_{2}$ of $\bar{X}$ have the property that $S_{i} \cap S_{i+1} \neq \varnothing$ for $i=1, \ldots, r-1$. Then apply the above case successively to $s_{i}$ and $s_{i+1}$.) Now, since $C_{1}$ and $C_{2}$ intersect, choose $y \in C_{1} \cap C_{2}$. Clearly, $\bar{X}$ is not smooth at $y$. Since $\bar{X} \backslash \bar{Z}$ is smooth, it follows that $y \in \bar{Z}$. Choose $y_{1}, y_{2} \in \bar{X}$ lying above $y$ such that $y_{1}$ belongs to $C_{1}$ and $y_{2}$ to $C_{2}$. By definition of $Y, y_{1}$ and $y_{2}$ belong to $\bar{Y}$ and the connected components to which they belong are denoted by the same letters. We then have $\phi\left(y_{1}\right)=x_{1}, \phi\left(y_{2}\right)=x_{2}$ and $\psi\left(y_{1}\right)=y=\psi\left(y_{2}\right)$. This proves the theorem.

\section{Acknowledgements}

We would like to thank the referee, whose valuable suggestions simplified and clarified many arguments and also improved the presentation of the paper as a whole. This paper is based on the author's master's thesis. The author would like to express his gratitude to his advisor Thomas Geisser, who suggested the topic of this paper. He would also like to thank Lars Hesselholt and Rin Sugiyama for their numerous helpful suggestions and comments in the course of writing his thesis. 


\section{References}

[Akhtar 2004] R. Akhtar, "Zero-cycles on varieties over finite fields", Comm. Algebra 32:1 (2004), 279-294. MR 2005b:14017 Zbl 1062.14013

[Bass and Tate 1973] H. Bass and J. Tate, "The Milnor ring of a global field", pp. 349-446 in Algebraic K-theory, II: "Classical" algebraic K-theory and connections with arithmetic (Seattle, WA, 1972), edited by H. Bass, Lecture Notes in Math. 342, Springer, Berlin, 1973. MR 56 \#449 Zbl 0299.12013

[Bloch 1986] S. Bloch, "Algebraic cycles and higher K-theory", Adv. in Math. 61:3 (1986), 267-304. MR 88f:18010 Zbl 0608.14004

[Geisser and Levine 2000] T. Geisser and M. Levine, "The $K$-theory of fields in characteristic $p$ ", Invent. Math. 139:3 (2000), 459-493. MR 2001f:19002 Zbl 0957.19003

[Geisser and Levine 2001] T. Geisser and M. Levine, "The Bloch-Kato conjecture and a theorem of Suslin-Voevodsky”, J. Reine Angew. Math. 530 (2001), 55-103. MR 2003a:14031 Zbl 1023.14003

[Harder 1977] G. Harder, "Die Kohomologie $S$-arithmetischer Gruppen über Funktionenkörpern”, Invent. Math. 42 (1977), 135-175. MR 57 \#12780 Zbl 0391.20036

[de Jong 1996] A. J. de Jong, "Smoothness, semi-stability and alterations", Inst. Hautes Études Sci. Publ. Math. 83 (1996), 51-93. MR 98e:14011 Zbl 0916.14005

[Kelly 2012] S. Kelly, Triangulated categories of motives in positive characteristic, Ph.D. thesis, Université Paris 13, 2012, Available at http://arxiv.org/abs/1305.5349.

[Kondo and Yasuda 2013] S. Kondo and S. Yasuda, "On two higher Chow groups of schemes over a finite field", 2013. arXiv 1306.1607

[Liu 2002] Q. Liu, Algebraic geometry and arithmetic curves, Oxford Graduate Texts in Mathematics 6, Oxford University Press, 2002. MR 2003g:14001 Zbl 0996.14005

[Mazza et al. 2006] C. Mazza, V. Voevodsky, and C. Weibel, Lecture notes on motivic cohomology, Clay Mathematics Monographs 2, American Mathematical Society, Providence, RI, 2006. MR 2007e:14035 Zbl 1115.14010

[Quillen 1972] D. Quillen, "On the cohomology and $K$-theory of the general linear groups over a finite field”, Ann. of Math. (2) 96 (1972), 552-586. MR 47 \#3565 Zbl 0249.18022

[Soulé 1979] C. Soulé, " $K$-théorie des anneaux d'entiers de corps de nombres et cohomologie étale", Invent. Math. 55:3 (1979), 251-295. MR 81i:12016 Zbl 0437.12008

[Voevodsky 2010] V. Voevodsky, "Cancellation theorem", Doc. Math. Extra volume: Andrei A. Suslin sixtieth birthday (2010), 671-685. MR 2012d:14035 Zbl 1202.14022

[Voevodsky et al. 2000] V. Voevodsky, A. Suslin, and E. M. Friedlander, Cycles, transfers, and motivic homology theories, Annals of Mathematics Studies 143, Princeton University Press, 2000. MR 2001d:14026 Zbl 1021.14006

Received 24 Dec 2014. Revised 1 Feb 2015. Accepted 15 Feb 2015.

TOHRU KOHRITA: kohrita.tohru@j .mbox.nagoya-u.ac.jp

Graduate School of Mathematics, Nagoya University, Nagoya, 464-8602, Japan 


\title{
ANNALS OF K-THEORY
}

\author{
msp.org/akt
}

EDITORIAL BOARD

\author{
Paul Balmer University of California, Los Angeles, USA \\ balmer@math.ucla.edu \\ Spencer Bloch University of Chicago, USA \\ bloch@math.uchicago.edu \\ Alain Connes Collège de France; Institut des Hautes Études Scientifiques; Ohio State University \\ alain@connes.org \\ Guillermo Cortiñas Universidad de Buenos Aires and CONICET, Argentina \\ gcorti@dm.uba.ar \\ Eric Friedlander University of Southern California, USA \\ ericmf@usc.edu \\ Max Karoubi Institut de Mathématiques de Jussieu - Paris Rive Gauche, France \\ max.karoubi@imj-prg.fr \\ Gennadi Kasparov Vanderbilt University, USA \\ gennadi.kasparov@vanderbilt.edu \\ Alexander Merkurjev \\ University of California, Los Angeles, USA \\ merkurev@math.ucla.edu \\ Amnon Neeman amnon.Australian National University \\ neeman@anu.edu.au \\ Jonathan Rosenberg (Managing Editor) \\ University of Maryland, USA \\ jmr@math.umd.edu \\ Marco Schlichting University of Warwick, UK \\ schlichting@warwick.ac.uk \\ Andrei Suslin Northwestern University, USA \\ suslin@math.northwestern.edu \\ Vladimir Voevodsky Institute for Advanced Studies, USA \\ vladimir@math.ias.edu \\ Charles Weibel (Managing Editor) \\ Rutgers University, USA \\ weibel@math.rutgers.edu \\ Guoliang Yu Texas A\&M University, USA \\ guoliangyu@math.tamu.edu \\ PRODUCTION \\ $\begin{array}{ll}\text { Silvio Levy } & \text { (Scientific Editor) } \\ \text { production@msp.org }\end{array}$
}

Annals of K-Theory is a journal of the K-Theory Foundation (ktheoryfoundation.org). The K-Theory Foundation acknowledges the precious support of Foundation Compositio Mathematica, whose help has been instrumental in the launch of the Annals of K-Theory.

See inside back cover or msp.org/ant for submission instructions.

The subscription price for 2016 is US $\$ 400 /$ year for the electronic version, and $\$ 450 /$ year (+\$25, if shipping outside the US) for print and electronic. Subscriptions, requests for back issues and changes of subscribers address should be sent to MSP.

Annals of K-Theory (ISSN 2379-1681 electronic, 2379-1683 printed) at Mathematical Sciences Publishers, 798 Evans Hall \#3840, c/o University of California, Berkeley, CA 94720-3840 is published continuously online. Periodical rate postage paid at Berkeley, CA 94704, and additional mailing offices.

AKT peer review and production are managed by EditFlow ${ }^{\circledR}$ from MSP.

PUBLISHED BY

mathematical sciences publishers nonprofit scientific publishing

http://msp.org/

(C) 2016 Mathematical Sciences Publishers 


\section{ANNALS OF K-THEORY}

2016

Statement of purpose

Jonathan M. Rosenberg and Charles A. Weibel

On the Deligne-Beilinson cohomology sheaves

Luca Barbieri-Viale

On some negative motivic homology groups

Tohru Kohrita

The joint spectral flow and localization of the indices of elliptic operators

Yosuke Kubota

A plethora of inertial products

Dan Edidin, Tyler J. Jarvis and Takashi Kimura 